\title{
First-Visit Diagnosis of Preperimetric Glaucoma
}

\author{
Avinoam Ophir ${ }^{*}$ \\ Division of Ophthalmology, Hillel-Yaffe Medical Centre, Hadera and The Ruth and Bruce Rappaport Faculty of \\ Medicine, The Technion, Haifa, Israel
}

\begin{abstract}
Purpose: To present a revised interpretation of the work-up data that enabled diagnosis of preperimetric glaucoma (PPG) at the first examination.

Methods: a) Literature analysis on PPG; b) 6-year follow-up of a glaucoma-suspect patient.

Results: Two new concepts may be adapted: (a) the objective finding of retinal nerve fiber layer (RNFL) thinning below the normal border in the opposing typical glaucoma locations, the inferior and superior quadrants, and in a non-diffuse pattern, appears asymptomatically and simultaneously only in glaucoma; and (b) the imaging-related RNFL thickness may be considered the reference glaucoma standard, whereas the suspicious early glaucomatous optic neuropathy, having a potential diagnostic inaccuracy, would serve as a complementary revealing finding. That approach enabled, in retrospect, a first-visit diagnosis of low-tension PPG in the patient. Diagnosis was confirmed after 6 years, when cecocentral scotoma and further RNFL thinning emerged despite treatment.
\end{abstract}

Conclusions: A revised approach enabled PPG diagnosis during the first visit.

Keywords: Preperimetric glaucoma, early glaucoma, low-tension glaucoma, optical coherence tomography, retinal nerve fiber layer.

\section{INTRODUCTION}

In glaucoma, structural injury has been documented to precede functional injury in most, but not all, eyes [1]. Recently, a change in the diagnostic criteria of glaucoma has been promoted such that glaucoma diagnosis may be made before the old prerequisites' functional criteria of standard automatic perimetry visual field (SAP- VF) loss are apparent, namely 'preperimetric glaucoma' (PPG). In PPG, structural injury, and especially glaucomatous optic neuropathy (GON), ought to be clinically proven. However, because of the inter-individual variability of the normal optic nerve head $(\mathrm{ONH})$, the subjective diagnosis of GON at its early stages, either clinically or by means of 3-D photos, is often challenging $[2,3]$. Detection of early loss of retinal nerve fibers, both clinically or by means of red-free photos, is also subjective and may be indecisive; more so in diffuse than in localized retinal nerve fibers loss [4]. In contrast, numerous studies have found the objective, quantitative assessment of retinal nerve fiber layer (RNFL) loss in early and advanced glaucoma by either optical coherence tomography (OCT, $-1,-2$ or -3 ) or scanning laser polarimetry (GDx-VCC) to be both accurate and reproducible [3-14]. In spite of these findings, the $\mathrm{ONH}$ evaluation is still considered the reference standard in PPG diagnosis, but proof of progressive worsening at the $\mathrm{ONH}$, the retinal nerve fibers or SAP-VF is required to verify diagnosis of early GON $[2,15]$. However, progression would naturally be coupled with further irreversible loss of RNFL and may occasionally be associated with VF injury.

*Address correspondence to this author at the Division of Ophthalmology, Hillel-Yaffe Medical Centre, Hadera, Israel; Tel: 04-6304458, Mobile: 0547222688; Fax: 03-5409222; E-mail: Ophthalmology@hy.health.gov.il
The OCT and GDx-VCC provide micron-resolution assessment of the peripapillary (3.4-mm in diameter) RNFL thickness. The RNFL loss in glaucoma, as depicted by these imaging devices, has been found to be directly associated with the severity of the disease [6-11]: In advanced glaucoma, RNFL loss is generally found to be significantly thinner than normal in all or most (except horizontal) sites. In moderate and early glaucoma associated with typical VF loss, peripapillary RNFL loss regularly occurs in typical locations, i.e., at the inferior and/or superior quadrants and/or globally. In eyes with PPG, in which SAP-VF is normal and $\mathrm{ONH}$ is determined as GON by other diagnostic modalities, such as the use of frequency-doubling technology, RNFL loss regularly occurs at the same typical locations as in perimetric glaucoma $[2,3,9,16]$.

Retinal nerve fiber layer thinning has been found to be a predictor of the development of glaucomatous visual field damage in glaucoma-suspect eyes, i.e., when the $\mathrm{ONH}$ looked clinically suspicious of being glaucomatous [17], but this group of authors stated that proof of progressive worsening of the disease is a prerequisite for diagnosing PPG, as mentioned above $[2,15]$. To avoid an irreversible disease progression in such eyes, in association with normal VF, it should be our goal to prompt or rule out a diagnosis of PPG. An interpretation of the cumulative data found during the evaluation of a 49 -year-old patient, which is based on a revised literature analysis, enabled a diagnosis of PPG at his first visit. To the best of my knowledge (search via Entrez Pubmed), this revised diagnostic approach is presented here for the first time.

\section{METHODS}

The study is based on analysis of the literature review on RNFL loss in association with perimetric glaucoma and PPG 
diagnosis. Conclusions refer to a revised interpretation of these cumulative data. That analysis was retrospectively carried out and a PPG diagnosis confirmed after 6 years of follow-up of a patient who presented initially with suspicious GON, low intraocular pressure, normal SAP-VF and abnormally thinned RNFL in each eye.

\section{RESULTS}

\section{A) Literature Analysis}

The cumulative data on the micron-resolution RNFL imaging studies, in association with the various stages of glaucoma, point out the following:

1. There is potential for inaccuracy in the subjective clinical determination of suspicious $\mathrm{ONH}$ at its early glaucoma stage as GON [2, 3, 8, 15];

2. Experts of the Optic Disk Reading Center found that even detection of GON progression is challenging if changes are subtle [17];

3. Looking for a proof of disease progression in order to define a glaucoma-suspect as having PPG might be risky. For example, complicated progression ensued in the presented patient when the foremost detectable progression was associated with cecocentral scotoma and reduction in visual acuity (see below);

4. On the other hand, the RNFL thickness assessment by high-resolution imaging devices is most often objective and accurate [5-16]. Several imagingrelated RNFL studies on PPG-suspect eyes overcame the obstacle of doubtful GON diagnosis by either (a) assessing eyes with abnormal doubling technology perimetry as glaucomatous (PPG) [10]; (b) studying the fellow eyes of glaucoma patients with unilateral VF loss [3]; or (c) determining GON only in eyes with documented evidence of progressive $\mathrm{ONH}$ changes [2]. In these PPG studies, significant RNFL loss emerged at the same locations as typically occur in the more advanced perimetric glaucoma, i.e., at the inferior quadrant, superior quadrant and/or globally $[2,3,9,16]$;

5. A comparative study has shown that an objective structural assessment of the RNFL thickness by the imaging devices is more accurate than clinical evaluation of disc photographs in PPG [3].

Based on these five points, a revised diagnostic approach of glaucoma at the preperimetric stage is considered such that the subjective clinical diagnosis of GON as the reference standard in diagnosing PPG would probably be changed by adopting the available and more accurate alternative: objective and quantitative assessment of the peripapillary RNFL thickness loss. This change may be undertaken by recognizing that three findings appear concurrently only in glaucoma: (a) the presence of suspicious GON; (b) in an asymptomatic patient; together with (c) simultaneous RNFL loss in a non-diffuse pattern at the two opposing locations, the superior and inferior quadrants (Fig. 1), without RNFL nasal quadrant attenuation, to a level below the lower border of the normative database (in OCT-2 and to the red area in OCT-3). These observations have not been reported (search via Entrez Pub-Med) in any RNFL study on other optic neuropathies, including multiple sclerosis, Parkinson's disease, ischemic optic neuropathy, retrobulbar neuritis, branch retinal artery occlusion, traumatic optic neuropathy, dominant optic atrophy, Leber's hereditary optic neuropathy (LHON) and AIDS optic neuropathy [18-28]. All of these are, by and large, symptomatic at presentation and/or present with diffuse RNFL thinning, rather than presenting as a loss at the two opposing, typical glaucomatous sites.

The revised interpretation presented here enabled, in retrospect, a first-visit diagnosis of low-tension PPG. The OCT initially disclosed bilateral typical RNFL thinning, and diagnosis was confirmed after 6 years, when cecocentral scotoma and further RNFL thinning emerged despite topical treatment.

\section{B) Case Report}

A 49-year-old healthy man was referred to the outpatient clinic of our institution because of bilateral suspicious GON ("glaucoma suspect") and normal standard VFs. The patient reported no history of trauma or family history of visual loss, and he had not received any medication. On examination in February 2002, his best-corrected visual acuity (BCVA) was 6/12 in each eye with myopic astigmatism; IOP was 12 $\mathrm{mmHg}$ in the RE and 14 in the LE, respectively, without hypotensive treatment. The anterior chamber angle was open +4 bilaterally. The ONHs had vertical cup/disc ratios of 0.7 and thin rims, and the optic cup in the right eye (RE) looked deeper than that in the other disk. An OCT examination (OCT-2000, Carl Zeiss Meditec, Dublin, CA, USA) was carried out. The Circle group program, 3.4-mm in diameter, was centered on the $\mathrm{ONH}$ for the assessment of the peripapillary RNFL. Results were based on the mean of five subsequent good scans per eye. Comparison was made in the graphic presentation with the age-matched OCT normative database (Fig. 1). The 24-2 Swedish Interactive Testing Algorithm of the SAP (Humphrey Zeiss Meditec, Dublin, CA, USA) was used for VF examination.

Quantitative, numerical analysis of the first OCT findings can be made at this time only with our own RNFL agematched normative database $(n=30$; Fig. 1) since numerical data of the OCT manufacturer normative database are not available. Furthermore, two instruments with the same software can have different RNFL-t outputs [3]. The mean global normal RNFL-t in our instrument is $138 \pm 30 \mu \mathrm{m}$, and its mean lower border is $113 \pm 31 \mu \mathrm{m}$. The range between the upper and lower borders in each site (i.e., clock-hour) in our normative database is thinner than that of the OCT manufacturer normative database.

In February 2002, the RNFL-t was bilaterally thinner than the lower border of the OCT- manufacturer normative database, mainly in the inferior quadrants, the superiortemporal site, the temporal quadrant and globally in each eye (Figs. 1, 2A). Quantitative comparison at that initial stage with our own normative database revealed thinning of the RNFL below its lower border in the two typical RNFL-loss glaucomatous locations by $37 \%$ (from 150 to $94 \mu \mathrm{m}$; Fig. 1) in the inferior quadrant and $43 \%$ in the superior-temporal site in the RE, and by $32 \%$ and $42 \%$ in the LE, respectively (Fig. 1). Mean global (4 quadrants) RNFL-t (Table at Fig. 2A) was $95 \mu \mathrm{m}$ in the RE and $104 \mu \mathrm{m}$ in the LE, which was lower by $16 \%$ and $8 \%$, respectively, than the mean RNFL-t 


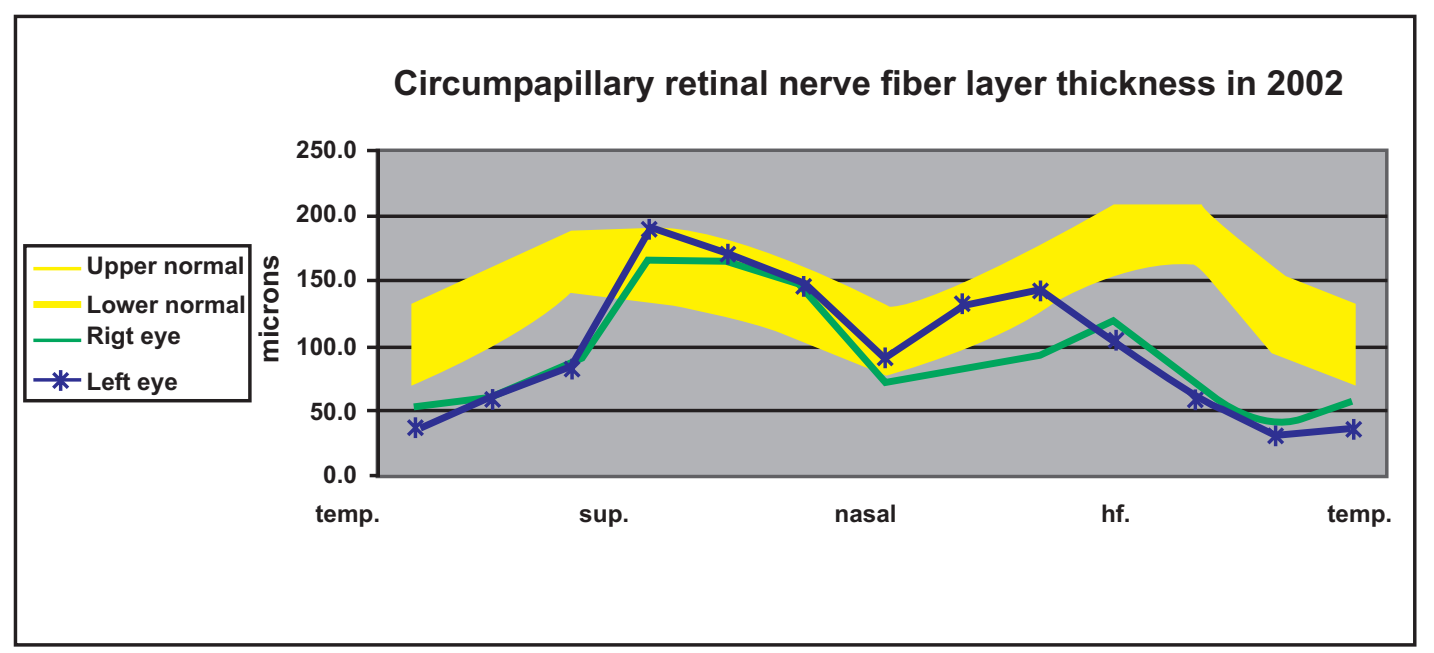

Fig. (1). Initial (in 2002) peripapillary retinal nerve fiber layer (RNFL) thickness as detected by the optical coherence tomography (OCT) in each eye of a 49-year-old patient with suspicious glaucomatous optic neuropathy ("glaucoma suspect"). Numeric comparison is made with the self-normative database software (bound in a double hump area).

lower border, $113 \mu \mathrm{m}$ (Fig. 1). The mean RNFL of only the temporal quadrant was markedly thinner than the mean of the lower border self-normative database, by $46 \%$ and $53 \%$, respectively. The VFs were full (Fig. 2).

A 24-hour diurnal-curve IOP took place thereafter, and IOPs ranged between 12-14 $\mathrm{mmHg}$ in each eye. Based on the clinical suspicion of early GON associated with RNFL thinning in two opposing typical glaucoma locations, i.e., in the inferior quadrant and the superior-temporal site, as well as globally, diagnosis of low-tension PPG was made. Col. betaxolol $0.5 \%$, a hypotensive and possibly neuroprotective agent, was prescribed b.i.d. following the diurnal curve examination for safety reasons.

According to the patient, during 6 years of follow-up, examinations were usually carried out in a community clinic every 3 to 4 months, while he was periodically referred for clinical and VF examinations. During follow-up, obstructive sleep apnea was ruled out per anamnesis. Central corneal thicknesses were 512 and $520 \mu \mathrm{m}$, for the $\mathrm{LE}$ and RE, respectively. The IOP ranged between $10-14 \mathrm{mmHg}$ in each eye under topical betaxolol, except once, when IOP was 20 $\mathrm{mmHg}$ in the RE. Visual fields were examined every 15-20 months and were full until and including February 2006. On the subsequent work-up in our clinic in September 2007, the cup/disc ratio was found to have worsened to 0.8 in each eye with thin rims, and cecocentral scotoma was found, mainly in the LE (Fig. 2B). Topical brimonidine tartrate $0.2 \%$ was bilaterally added. After 8 months, the scotoma widened in the RE (Fig. 2C) and BCVA was 6/18 in that eye; the scotoma in the LE became non-specific and more diffuse, while BCVA remained 6/12.

An OCT-RNFL evaluation was carried out for the second time in May 2008. Further RNFL thinning was found in the inferior $(26 \%)$ and temporal $(67 \%)$ quadrants and globally (29\%) of the RE, while it occurred in the superior quadrant $(14 \%)$ in the LE. In addition, further RNFL thinning was detected bilaterally at various other sites (Fig. 2B). A third OCT examination was done four months later, revealing similar data. The IOP in a repeat diurnal curve ranged between 9-14 $\mathrm{mmHg}$ in each eye and was followed later by further worsening of the cecocentral scotoma thinning in the RE (not presented). Approval of the local Ethics Committee was not required for this report.

\section{DISCUSSION}

Based on a revised deduction from the literature review, the objective finding of a simultaneous RNFL thinning in two typical glaucoma locations that appeared asymptomatically and in the presence of suspicious early GON in the presented patient raised the possibility of PPG diagnosis during the first work-up visit. After 6 years, diagnosis was confirmed when progression of the disease had been found, manifesting in the emergence of cecocentral scotoma, bilateral worsening of GON and further RNFL thinning, despite topical treatment. This revised deduction may be supported by the following:

Studies have suggested that RNFL assessment may become a stand-alone criterion for diagnosing PPG. Lu et al. stated that the best stand-alone diagnostic strategy for OCT RNFL data is to classify an eye as glaucomatous if the combination of the overall, inferior quadrant and superior quadrant RNFL thickness average is below normal [8]. In regard to RNFL loss vs. function relationship, Horn et al. found a clear association of the inferior and superior RNFL loss with the corresponding area of VF loss [10]. Hood et al. suggested that hemifield may be considered glaucomatous if one of the 5 clock-hour RNFL sectors ( 3 and 9 excluded) is below the normative database, i.e., at $<1 \%$ area (red area of the OCT-3 output graph), or two are at $<5 \%$ (yellow) [11]. In that regard, Caprioli et al. found that OCT is superior to both GDx-VCC and HRT II in its ability to detect RNFL loss in glaucoma [3]. The presented diagnostic interpretation is in accordance with these studies. However, several studies have shown that none of the new imaging instruments has a perfect diagnostic accuracy or is flawless. It is not unusual to find false positives or cases detected as abnormal by the imaging instruments and not confirmed by more conventional methods. 

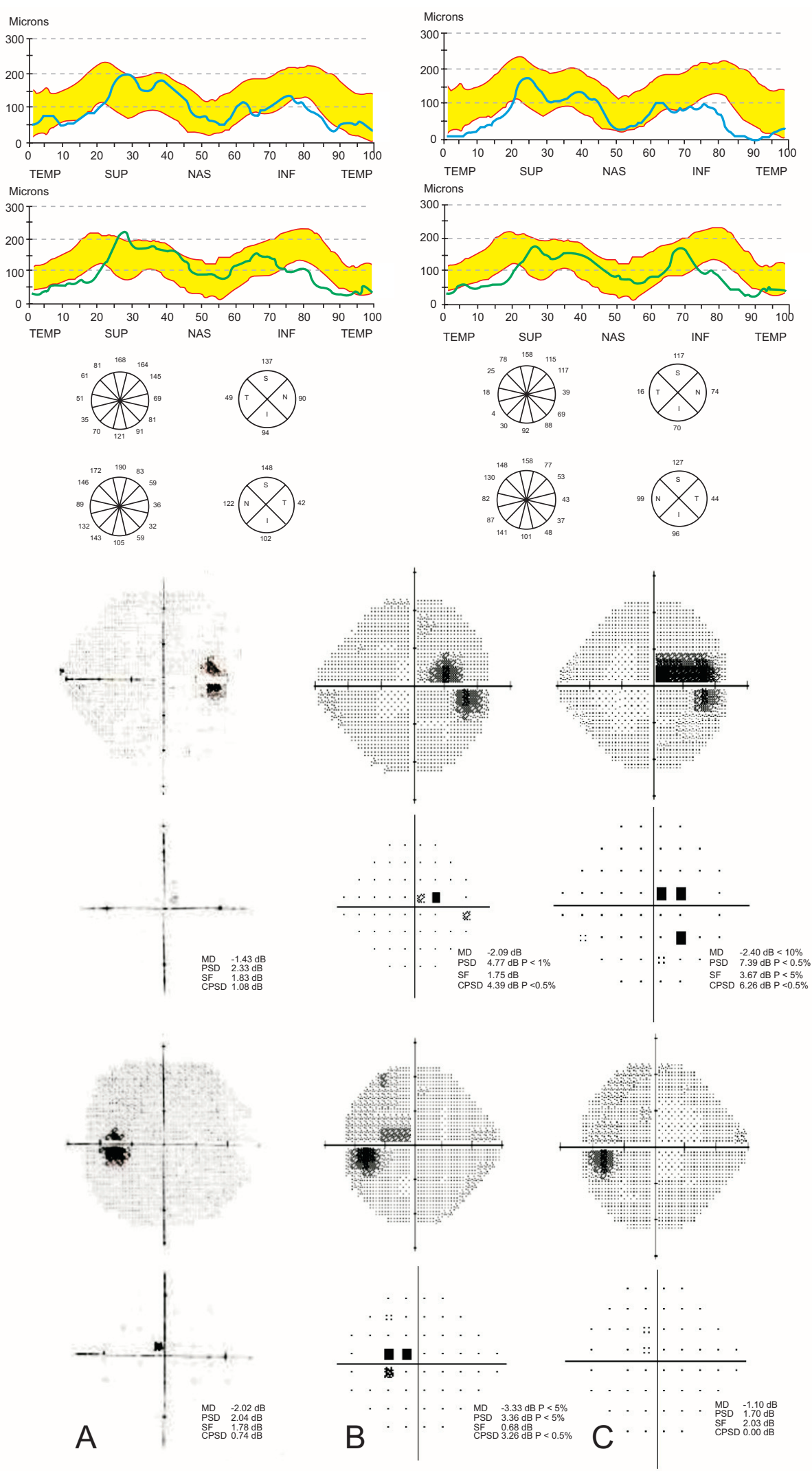

Fig. (2). (A) Top: The mean initial OCT RNFL examination of 5 scans is presented in clock-hours, quadrants, and graphically, in comparison with the OCT age-matched normative database software provided by the manufacturer (bound in yellow). The RNFL is thinner than the lower normal border in both the right (OD) and left eye (OS) superiorly, inferiorly and temporally. Bottom: Standard visual fields are normal. (B) Visual fields in September 2007. Cecocentral scotoma is evident bilaterally, more in the left eye. (C). Top: In May 2008, further thinning of the RNFL is detected in the inferior, superior and temporal quadrants of the right eye and in the superior quadrant of the left eye. Bottom: The cecocentral scotoma widened in the right eye and became non-specific and less distinct in the left eye. 
We used the OCT-2 instrument in our study. Its reproducibility between examinations in perimetric glaucoma was found to be $11.8 \%$ [5]. The aforementioned PPG studies used OCT-3 or GDx-VCC [2, 3, 9, 16]. The output of RNFL thickness in OCT-3 is parallel to that of OCT-2, although RNFL thickness data in the OCT-2 are generally thicker [14]. We assume that further studies would quantitatively clarify (a) at which level a localized RNFL loss should be considered pathological and (b) the minimum required area, quadrants or sites of substantial RNFL thinning, including mean global loss, for designating the eye as glaucomatous.

In addition to disease progression, a clinical aid in diagnosing PPG may be the frequency-doubling technology or short-wavelength automated perimetry, although RNFL loss commonly precedes VF loss as detected by these perimeters $[3,16]$. In the case of diffuse RNFL thinning, awareness ought to be raised to the possibility that RNFL thickness may vary significantly with age, ethnicity, axial length, and optic disc area [29] or may be reduced as a result of low signal strength. In contrast, in the presented patient, RNFL thinning was not diffuse but attained a localized pattern in a few locations.

The presence of risk factors for glaucoma, such as increased IOP, did not play a role in determining PPG diagnosis in any of the aforementioned studies $[2,3,9,16]$, probably because real-time status should be assessed at the time of examination, rather than to predict future possible risks. In contrast, once diagnosis of glaucoma has been established, treatment to reduce IOP to the pre-determined target IOP is well accepted.

In summary, a revised interpretation of the data in the diagnosis of PPG is presented. That approach enabled, in retrospect, a diagnosis of PPG in an adult during the first visit, which was verified 6 years later. It was based on the literature findings that the pattern of RNFL-t loss at the typical glaucomatous sites was repeatedly found to reflect glaucoma, including its preperimetric stage. More importantly, the OCT affords an objective and accurate realtime structural state of the RNFL-t that indicates whether RNFL loss at a specific glaucomatous site has already occurred in the glaucoma suspect. Further studies are required to confirm the presented interpretation of RNFL data and to provide quantitative criteria for early diagnosis of PPG.

\section{REFERENCES}

[1] Sommer A, Katz J, Quigley HA, Miller NR, Robin AL, Richter RC. Clinically detectable nerve fiber atrophy precedes the onset of glaucomatous field loss. Arch Ophthalmol 1991; 109: 77-83.

[2] Medeiros FA, Zangwill LM, Bowd C, Sample PA, Weinreb RN. Use of progressive glaucomatous optic disk change as the reference standard for evaluation of diagnostic tests in glaucoma. Am J Ophthalmol 2005; 139: 1010-8.

[3] Caprioli J, Nouri-Mahdavi K, Law SK, Badalà F. Optic disc imaging in perimetrically normal eyes of glaucoma patients with unilateral field loss. Trans Am Ophthalmol Soc 2006; 104: 202-11.

[4] Airaksinen PJ, Tuulonen A, Werner EB. Clinical evaluation of the optic disc and retinal nerve fiber layer. In: Ritch R, Shields MB, Krupin T, Eds. The Glaucomas. St. Louis: Mosby; 1996: 617-57.

[5] Blumenthal EZ, Williams JM, Weinreb RN, Girkin CA, Berry CC, Zangwill LM. Reproducibility of nerve fiber layer thickness measurements by use of optical coherence tomography. Ophthalmology 2000; 107: 2278-82.
[6] Kanamori A, Nakamura M, Escano MF, Seya R, Maeda H, Negi A. Evaluation of the glaucomatous damage on retinal nerve fiber layer thickness measured by optical coherence tomography. Am J Ophthalmol 2003; 135: 513-20.

[7] Sihota R, Sony P, Gupta V, Dada T, Singh R. Diagnostic capability of optical coherence tomography in evaluating the degree of glaucomatous retinal nerve fiber damage. Invest Ophthalmol Vis Sci 2006; 47: 2006-10.

[8] Lu AT, Wang M, Varma R, Schuman JS, Greenfield DS, Smith SD. Combining nerve fiber layer parameters to optimize glaucoma diagnosis with optical coherence tomography. Ophthalmology 2008; 115: 1352-7.

[9] Bagga H, Feuer WJ, Greenfield DS. Detection of psychophysical and structural injury in eyes with glaucomatous optic neuropathy and normal standard automated perimetry. Arch Ophthalmol 2006; 124: 169-76.

[10] Horn FK, Mardin CY, Viestenz A, Jünemann AG. Association between localized visual field losses and thickness deviation of the nerve fiber layer in glaucoma. J Glaucoma 2005; 14: 419-25.

[11] Hood DC, Harizman N, Kanadani FN, Grippo TM, Baharestani S Greenstein VC. Retinal nerve fibre thickness measured with optical coherence tomography accurately detects confirmed glaucomatous damage. Br J Ophthalmol 2007; 91: 905-7.

[12] Kim TW, Park UC, Park KH, Kim DM. Ability of Stratus OCT to identify localized retinal nerve fiber layer defects in patients with normal standard automated perimetry results. Invest Ophthalmol Vis Sci 2007; 48: 1635-41.

[13] Sehi M, Guaqueta DC, Feuer WJ, Greenfield DS. A comparison of structural measurements using 2 Stratus optical coherence tomography instruments. J Glaucoma 2007; 16: 287-92.

[14] Bourne RR, Medeiros FA, Bowd C, Jahanbakhsh K, Zangwill LM, Weinreb RN. Comparability of retinal nerve fiber layer thickness measurements of optical coherence tomography instruments. Invest Ophthalmol Vis Sci 2005; 46: 1280-5.

[15] Greenfield DS, Weinreb RN. Role of optic nerve imaging in glaucoma clinical practice and clinical trials. Am J Ophthalmol 2008; 145: 598-603.

[16] Brusini P, Zeppieri M, Tosoni C, Parisi L, Felletti M, Salvetat ML. Stratus-OCT imaging in early glaucomatous and in ocular hypertensive patients with and without frequency-doubling technology abnormalities. Eye 2008; 22: 406-13.

[17] Mohammadi K, Bowd C, Weinreb RN, Medeiros FA, Sample PA, Zangwill LM. Retinal nerve fiber layer thickness measurements with scanning laser polarimetry predict glaucomatous visual field loss Am J Ophthalmol 2004; 138: 592-601.

[18] Parrish RK 2nd, Schiffman JC, Feuer WJ, Anderson DR, Budenz DL, Wells-Albornoz MC. Test-retest reproducibility of optic disk deterioration detected from stereophotographs by masked graders. Am J Ophthalmol 2005; 140: 762-4.

[19] Henderson AP, Trip SA, Schlottmann PG, Altmann DR, GarwayHeath DF, Plant GT. An investigation of the retinal nerve fibre layer in progressive multiple sclerosis using optical coherence tomography. Brain 2008; 131: 277-87.

[20] Inzelberg R, Ramirez JA, Nisipeanu P, Ophir A. Retinal nerve fiber layer thinning in Parkinson disease. Vision Res 2004; 44: 2793-7.

[21] Hood DC, Anderson S, Rouleau J, Wenick AS, Grover LK, Behrens MM. Retinal nerve fiber structure versus visual field function in patients with ischemic optic neuropathy. A test of a linear model. Ophthalmology 2008; 115: 904-10.

[22] Pro MJ, Pons ME, Liebmann JM, et al. Imaging of the optic disc and retinal nerve fiber layer in acute optic neuritis. J Neurol Sci 2006; 250: 114-9.

[23] Asefzadeh B, Ninyo K. Longitudinal analysis of retinal changes after branch retinal artery occlusion using optical coherence tomography. Optometry 2008; 79: 85-9.

[24] Medeiros FA, Moura FC, Vessani RM, Susanna R Jr. Axonal loss after traumatic optic neuropathy documented by optical coherence tomography. Am J Ophthalmol 2003; 135: 406-8.

[25] Kim TW, Hwang JM. Stratus OCT in dominant optic atrophy: features differentiating it from glaucoma. J Glaucoma 2007; 16: 655-8.

[26] Barboni P, Savini G, Valentino ML, Montagna P, Cortelli P, De Negri AM. Retinal nerve fiber layer evaluation by optical coherence tomography in Leber's hereditary optic neuropathy. Ophthalmology 2005; 112: 120-6. 
[27] Bellusci C, Savini G, Carbonelli M, Carreli V, Sadun AA, Barboni $P$. Retinal nerve fiber layer thickness in nonarteritic anterior ischemic optic neuropathy: OCT characterization of the acute and resolving phases. Graefe's Arch Clin Exp Ophthalmol 2008; 246: 641-7.
[28] Seo JH, Hwang JM, Park SS. Comparison of retinal nerve fibre layers between 11778 and 14484 mutations in Leber's hereditary optic neuropathy. Eye 2009; 24: 107-11.

[29] Budenz DL, Anderson DR, Varma R, Schuman J, Cantor L, Savell J. Determinants of normal retinal nerve fiber layer thickness measured by Stratus OCT. Ophthalmology 2007; 114: 1046-52.

(C) Avinoam Ophir; Licensee Bentham Open.

This is an open access article licensed under the terms of the Creative Commons Attribution Non-Commercial License (http: //creativecommons.org/licenses/by$\mathrm{nc} / 3.0 /$ ) which permits unrestricted, non-commercial use, distribution and reproduction in any medium, provided the work is properly cited. 